\title{
Preparation of Microcrystalline Cellulose from Water Hyacinth Reinforced Polylactic Acid Biocomposite
}

\author{
Teerapa Semachai ${ }^{1}$, Panitnad Chandranupap ${ }^{1}$ and Pravitra Chandranupap ${ }^{2}$ \\ 1 Department of Chemical Engineering, Faculty of Engineering, \\ King Mongkut's University of Technology North Bangkok, Thailand \\ ${ }^{2}$ Department of Industrial Chemistry, Faculty of Applied Science, \\ King Mongkut's University of Technology North Bangkok, Thailand
}

\begin{abstract}
In this work, we successfully mixed polylactic acid (PLA) with microcrystalline cellulose (MCC) from water hyacinth. The MCC was prepared by treating water hyacinth fiber (WHF). Then hydrochloric acid was used to hydrolyze treated fiber to MCC. X-rays diffraction (XRD) showed that the MCC produced has 73.28 per cent crystallinity. Internal mixing was used to combine composites between MCC and PLA. Percentages of MCC were 1, 5, 10 and 15, respectively. Fourier transform infrared (FT-IR) spectroscopy indicated that the interaction between MCC and PLA are only mechanically interaction. Tensile testing of this composite (ASTM D638) revealed that tensile strength and percentage of elongation at break decreased but the increase of young's modulus. The morphological analysis was observed thru composites fractured surface by Scanning Electron Microscope (SEM). They showed a void between cellulosic fiber and PLA when high amount of MCC conformed with tensile results.
\end{abstract}

\section{Introduction}

Nowadays, there is an increasing demand for products made from renewable and sustainable non-petroleum based resources. Biopolymer-based materials have been interested because they are environmental friendly materials [1].

Polylactic acid (PLA) is the second most important bioplastic of the world in regard to consumption in volume [2]. It has been used in several applications such as food packaging, water and milk bottles, barriers for sanitary products and diapers, as well as in automotive applications [3]. Its can be derived from renewable resources like starch so it has high water vapor permeability but poor mechanical properties. To improve such properties, some additives or other ingredients such as nanoclays, fibers, carboxymathylcellulose (CMC), microcrystalline cellulose (MCC), etc. have been added [4].

Microcrystalline cellulose (MCC) is native from cellulose, which can be found in wood pulp. The cellulose polymers in pulp are reduced to small chain polymers or microcrystals by hydrolysis. Their application in composite materials has attracted attention due to their high mechanical strength, high stiffness, renewability, biodegradability and low weight. MCC is characterized by a high degree of crystallinity, and the values typically range between $55 \%$ and $80 \%$ [5]. Lignocellulosic material was generally used to produce MCC such as kenaf fiber, cotton, oil palm biomass, etc.
Water hyacinth is an aquatic plant which can live and reproduce floating freely. It became dense coverage that prevents the sunlight from penetrating the water due to very fast-growing plant. The weed removes oxygen from the water, resulting in the death of many fish. Many countries, including Thailand, try to convert this plant into valuable things, such as material for papermaking, charcoal briquetting and etc. [6]. The aim of this research is to prepare MCC from water hyacinth and study the effect on morphological, thermal and mechanical properties of PLA-MCC composite.

\section{Experimental}

\subsection{Materials}

Polylactic acid (Nature Work TM PLA 2003D) was purchased from $\mathrm{BC}$ polymer marketing Co. Ltd. It has a specific gravity of $1.24 \mathrm{~g} / \mathrm{cm} 3$ and melt flow index (MFI) of $6 \mathrm{~g} / 10 \mathrm{~min}\left(210^{\circ} \mathrm{C}, 2.16 \mathrm{~kg}\right)$. Water hyacinth fiber was purchased from Water hyacinth handicraft products group (Phayao, Thailand).

\subsection{Preparation of MCC}

Water hyacinth fiber (WHF) was soaked in sodium hydroxide solution $(10 \% \mathrm{w} / \mathrm{v}$ and $15 \% \mathrm{w} / \mathrm{v})$ for 1 hour at $60^{\circ} \mathrm{C}$ then washed until neutral and dried it in oven. $2 \mathrm{M}$ Hydrochloric acid was used to treat fiber for 6 hours at 
$80^{\circ} \mathrm{C}$. Hydrolyzed fiber was then washed and sieved to collect size below $100 \mu \mathrm{m}$. The hydrolyzed fiber was coded as MCC10 (or MCC15, depended on $\mathrm{NaOH}$ condition).

\subsection{Preparation of PLA and PLA/MCC composite}

About $59.4 \mathrm{~g} \mathrm{(57} \mathrm{g,} 54 \mathrm{~g}$ and $51 \mathrm{~g}$ ) of PLA pellets were

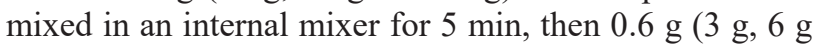
and $9 \mathrm{~g}$ ) of MCC were added. PLA and MCC were blended at $175^{\circ} \mathrm{C}$ until $15 \mathrm{~min}$ of process, mechanically stirred up to $38 \mathrm{rpm}$. The obtained mixture was coded as PLA/MCC1 (PLA/MCC5, PLA/MCC10 and PLA/MCC $15)$.

\subsection{Sample preparation}

Around $30 \mathrm{~g}$ of neat PLA and PLA composites were compression molded in a heated press at $180^{\circ} \mathrm{C}$ for $5 \mathrm{~min}$ without pressure. Then a pressure of 400 bar was applied for $5 \mathrm{~min}$. After this period, the pressure was release and the press plates were cooled below $60^{\circ} \mathrm{C}$ by cold water. Finally, the mold was removed from plates and sample sheet (1.2 mm thickness) was obtained.

\subsection{Characterization}

\subsubsection{X-ray diffraction $(X R D)$}

The XRD data were collected using an X-Ray diffractrometer (BRUKER model D8 Advance, Germany) equipped with $\mathrm{Cu}$ radiation at $40 \mathrm{kV}$ and 30 $\mathrm{mA}$ to investigate the XRD spectra of the cellulosic sample. Scattered radiation was detected in the range $2 \theta$ $=5-80^{\circ}$. A scanning step of $0.02^{\circ}$ is used at a time of 1 sec per step.

\subsubsection{Differential scanning calorimetry (DSC)}

Differential scanning calorimeter (Mettler Toledo TGA/DSC 1, USA) was used to study thermal behavior of PLA composite. The samples were heated from $30^{\circ} \mathrm{C}$ to $190^{\circ} \mathrm{C}$ at a heating rate of $10^{\circ} \mathrm{C} / \mathrm{min}$ under nitrogen atmosphere.

\subsubsection{Fourier-transform infrared (FT-IR) spectroscopy}

The FT-IR spectra of the cellulosic samples were recorded using a FT-IR Spectrometer (Perkin Elmer Spectrum 2000, USA) with a resolution of $4 \mathrm{~cm}^{-1}$ and within the wave number range $4000-400 \mathrm{~cm}^{-1}$.

\subsubsection{Tensile testing}

The test specimens of sample for tensile according to ASTM D638 type 5. Dog-bone shaped composite samples were cut by using a computer numerical control (CNC) machine. The tensile tests were operated using a universal testing machine (Testometric M500-25AT, UK), with a $10 \mathrm{kN}$ load cell with $1 \mathrm{~mm} / \mathrm{min}$ crosshead speed.
At least five samples were tested for each material and average values are presented.

\subsubsection{Scanning electron microscope (SEM)}

The Scanning Electron Microscope (SEM) was used to study morphology of MCC composites. Samples were sputter-coated with gold (Sputter coater, SPI Supplies, USA) to enhance the electrical conductivity. SEM was conducted using a JEOL JSM-6480LV model, a magnification of $300 \times$ and $1000 \times$ were applied.

\section{Results \& discussion}

\subsection{X-ray diffraction}

The crystallinity of MCC has been analyzed by x-ray diffractometer. Figure 1 showed that both MCC10 and MCC15 diffractograms showed only one outstanding peak around $2 \theta=22.5^{\circ}$ (for 200 plane). That is the characteristic of cellulose.

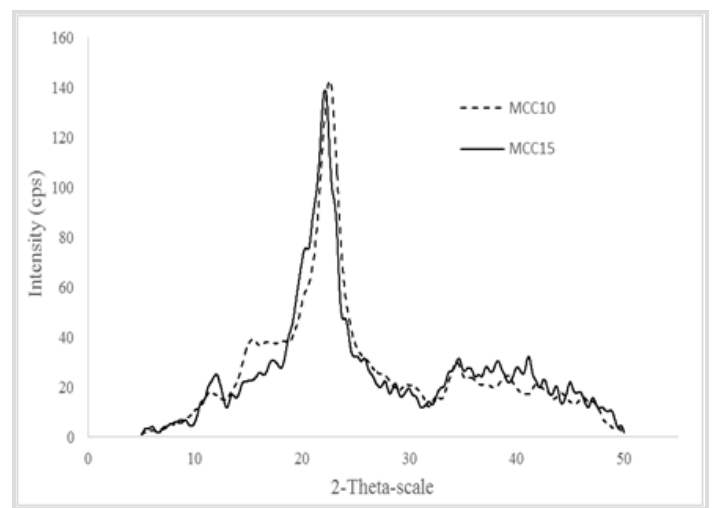

Figure 1. XRD of WHF treated with sodium hydroxide and hydrochloric acid.

The crystallinity $\left(\mathrm{I}_{\mathrm{C}}\right)$ was determined by means of equation (1) using the height of 200 peak $\left(\mathrm{I}_{2} 00,2 \theta=\right.$ $22.5^{\circ}$ ) and the minimum between the 200 and 110 peaks $\left(\mathrm{I}_{a m}, 2 \theta=18^{\circ}\right) . \mathrm{I}_{2} 00$ stands for both crystalline and amorphous material while $\mathrm{I}_{a m}$ stands for amorphous material [7].

$$
I_{C}=\left[\frac{I_{200}-I_{\operatorname{am}}}{I_{200}}\right] \times 100
$$

The synthesis cellulosic has $\mathrm{I}_{\mathrm{C}}$ around $73.28 \%$ and $76.44 \%$ for MCC10 and MCC15. The high percentage of crystallinity in confirmed that they are MCC.

\subsection{Differential scanning calorimetry}

Thermograph from DSC displays in Figure 2, neat PLA has a single melt peak, however with the addition of MCC showed a double peak forms. This double peak can be attributed to the coexistence of two crystalline populations or re-crystallization [8].

Table 1 showed the thermal properties of MCC composite glass temperature, crystallization temperature 
and melting temperature. It was remarkable to note that crystallization temperatures are decrease compared to neat PLA with the MCC addition into polymer matrix. Suggesting that the amount of MCC did not affect to glass and melting temperature of composites. Therefore it cannot be a nucleating agent due to less amount of MCC.

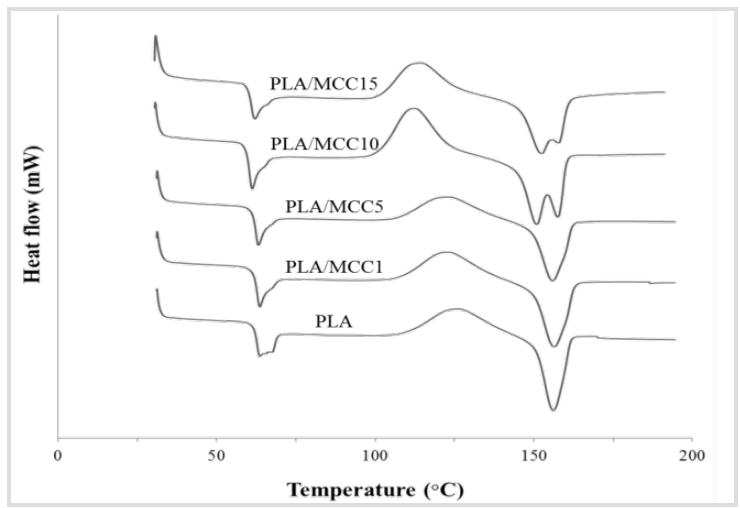

Figure 2. DSC thermographs of neat PLA and PLA/modified cellulose composites.

Table 1. Results from DSC.

\begin{tabular}{|l|c|c|c|}
\hline & $\mathbf{T}_{\mathbf{g}}\left({ }^{\circ} \mathbf{C}\right)$ & $\mathbf{T}_{\mathbf{c c}}\left({ }^{\circ} \mathbf{C}\right)$ & $\mathbf{T}_{\mathbf{m}}\left({ }^{\circ} \mathbf{C}\right)$ \\
\hline PLA & 59.46 & 121.54 & 150.35 \\
\hline PLA/MCC1 & 60.54 & 118.39 & 150.65 \\
\hline PLA/MCC5 & 59.07 & 118.53 & 150.00 \\
\hline PLA/MCC10 & 59.20 & 109.98 & 148.77 \\
\hline PLA/MCC15 & 59.97 & 111.89 & 149.07 \\
\hline
\end{tabular}

\subsection{Fourier transforms infrared spectroscopy}

The FT-IR analysis has been widely used to identify the interaction and phase behavior of polymer composites [10]. The FT-IR spectra of PLA and PLA/MCC composites were shown in Figure 3. Figure 3a exhibited characteristic spectrum of neat PLA. The broad band at $3330-3600 \mathrm{~cm}^{-1}$ is $\mathrm{O}-\mathrm{H}$ bending and starching for PLA. The peaks originated from the asymmetric stretching vibration of C-H are situated at 2880 to $2999 \mathrm{~cm}^{-1}$.

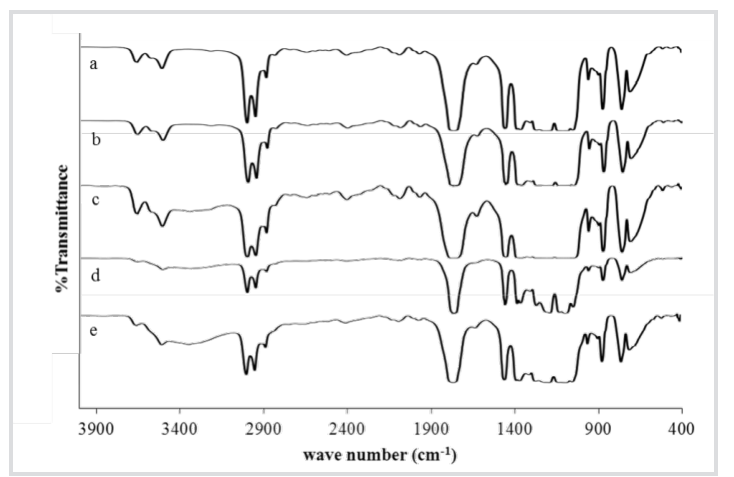

Figure 3. FT-IR spectra of PLA and PLA/MCC composites. They display (a) neat PLA (b) PLA/MCC1 (c) PLA/MCC5 (d) PLA/MCC10 and (e) PLA/MCC15.

The C-O stretching vibration of carbonyl is apparent at $1760 \mathrm{~cm}^{-1}$ and the peaks corresponding to $\mathrm{C}-\mathrm{H}$ deformation in the $\mathrm{CH}_{3}$ appeared at $1450 \mathrm{~cm}^{-1}$ [11].

Figure $3 \mathrm{~b}-3 \mathrm{e}$ showed the character peaks of PLA/MCC composites. They did not present any new peak compared to neat PLA. This result suggested that MCC and PLA composites were produced from physically interaction rather than chemical interaction [9].

\subsection{Tensile properties}

The trend of tensile properties of PLA and PLA/ MCC composites was shown in Figure 4.

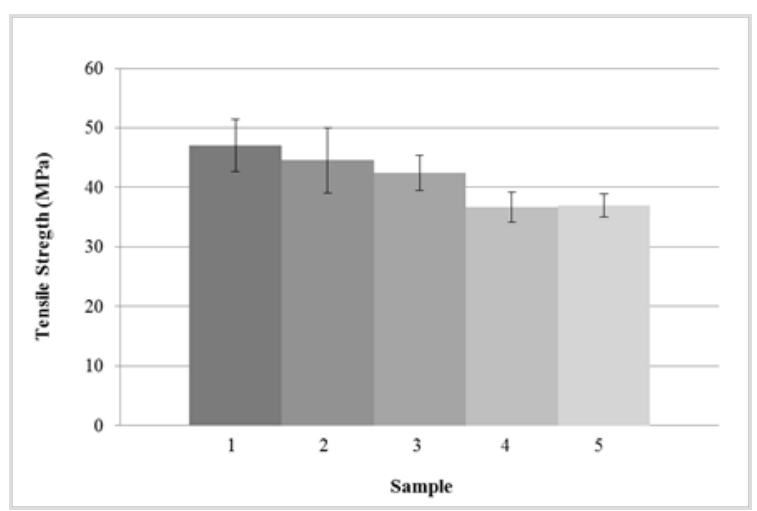

(a) Tensile strength

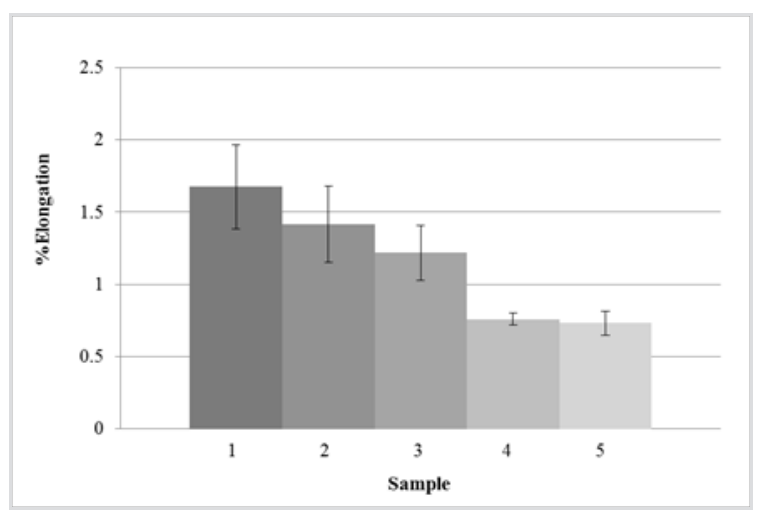

(b) Percentage of Elongation

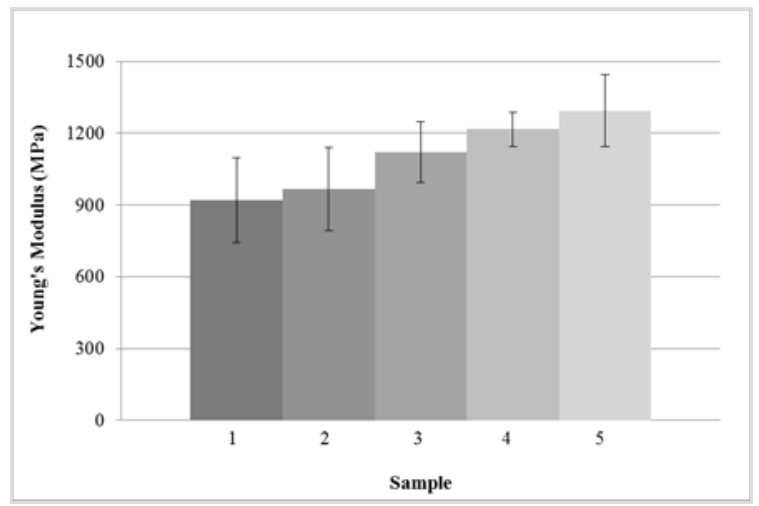

(c) Young's Modulus 
Figure 4. Tensile properties of PLA and PLA/MCC composites include (a) Tensile strength (b) Percentage of Elongation and (c) Young's Modulus, the samples are (1) neat PLA (2) PLA/ MCC1 (3) PLA/MCC5 (4) PLA/MCC10 and (5) PLA/MCC15.

From the result in Figure 6, addition of $\mathrm{MCC}$ into PLA matrix did not reveal any improvements in tensile strength and elongation at break when compared to neat PLA. In the other hand, the Young's modulus increased from 919 To $1293 \mathrm{MPa}$ with the increase of MCC loading. This is due to more hydrogen bonding, stiffening effect and high crystallinity index of the filler which is a characteristic of filler/polymer composite [11]. The aggregation of MCC may cause low tensile strength and elongation at break for composites due to Van der Waal's forces [12]. Because of this phenomenon, the filler-filler interaction becomes more effect than filler-matrix interaction. So they are poor interfacial adhesion between MCC and matrix, numerous voids can be generated at filler matrix interface as a result in worse tensile properties than neat PLA.

\subsection{Scanning electron microscope}

The cross-sectional surface of composites was used to observe distribution of MCC and study the interaction between two components. Small amount of cellulose was distributed into PLA matrix can be seen in Figure 5a-5b. They had a rougher surface compared to high percentage of MCC (Figure 5c-5d).
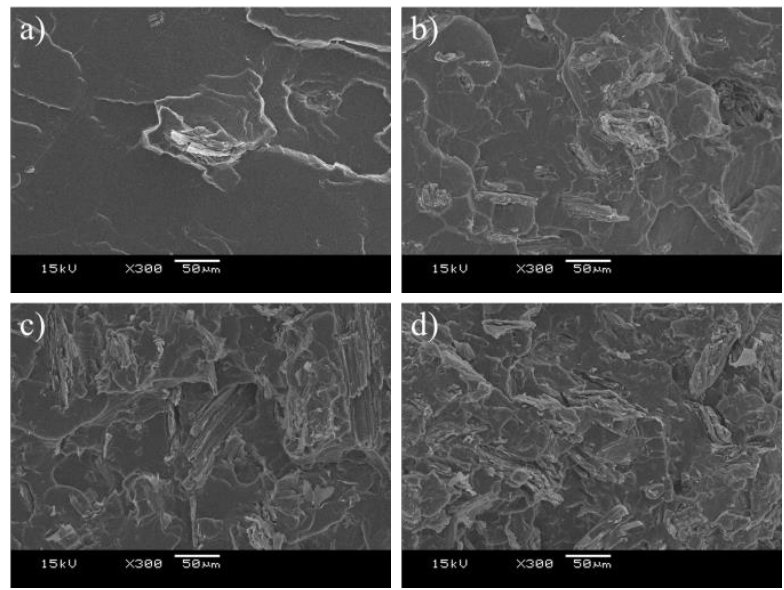

Figure 5. Scanning Electron Micrograph conducted with a magnification $300 \times$. They exhibit (a) PLA/MCC1 (b) PLA/ MCC5 (c) PLA/MCC10 and (d) PLA/MCC15.

MCC agglomeration appeared to occur with increased filler. More magnifying power demonstrated that they protruded out of fractured surface as shown in Figure 6c$6 \mathrm{~d}$ and also visible a void between cellulosic fiber and PLA. For these reasons indicate that the dispersion of MCC was not uniform and presented poor interfacial adhesion between filler and matrix [2, 9, and 11]. These can be the explanation for the tensile results that tensile strength and elongation at break decreased when increasing amount of MCC.
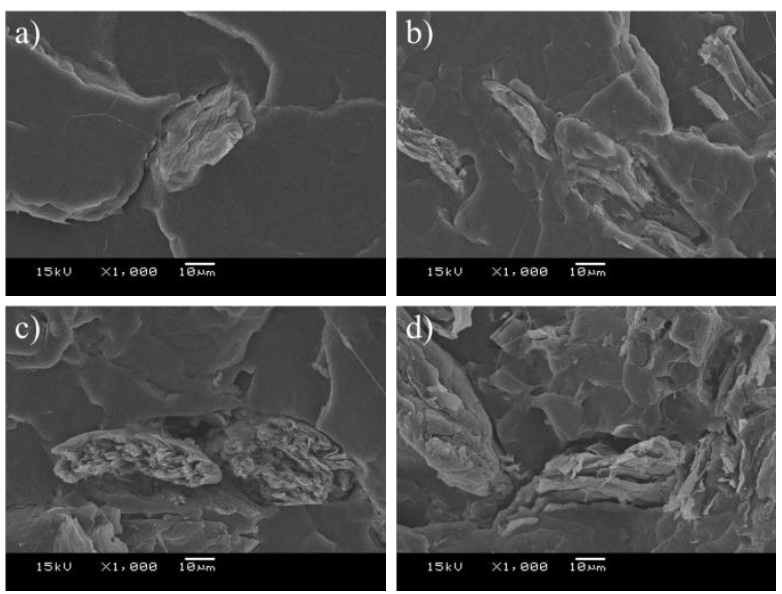

Figure 6. SEM image conducted with a magnification 1000× pictured of (a) PLA/MCC1 (b) PLA/ MCC5 (c) PLA/MCC10 and (d) PLA /MCC15.

\section{Conclusions}

MCC was successfully produced from water hyacinth and also mixed with PLA by internal mixing machine. The FT-IR spectra of PLA/MCC composites show no significant changes in the peaks position suggesting that only physical interaction between PLA and MCC occurred. Tensile properties demonstrate increasing of Young's modulus due to increase in hydrogen bonding and stiffening effect. In the other hand both of tensile strength and elongation at break decreased with increasing MCC loading because of a void between MCC and PLA resulted to poor interfacial adhesion. The mechanical properties may be further improved by using suitable coupling agents. This is expected to better tensile properties of composites.

\section{References}

1. M. Avella, G. B. Gaceva, A. Bužarovska, M. E. Errico, G. Gentile, A. Grozdanov. Poly (lactic acid)Based Biocomposites Reinforced with Kenaf Fibers. Journal of Applied Polymer science. 108, 3542-3551 (2008)

2. S. Joshi, U. Sharma, G. Goswami. Bio-Plastic from Waste Newspaper. International Conference on Emerging Trends of Research in Applied Sciences and Computational Techniques (2014)

3. M. Jonoobi, J. Harun, A. P.Mathew, K. Oksman. Mechanical properties of cellulose nano-fiber (CNF) reinforced polylactic acid (PLA) prepared by twin screw extrusion. Composites Science and Technology. 70, 1742-1747 (2010)

4. Y. Byun, Y. T. Kim. Chapter 14 - Bioplastics for Food Packaging: Chemistry and Physics. Innovations in Food Packaging (Second Edition) 353-368 (2014)

5. D. Trache, M. H. Hussin, C. T. Hui Chuin, S. Sabar, M.R. Fazita, O. F. Taiwo, T.M. Hassan, M.K. M. Haafiz. Microcrystalline cellulose: Isolation, characterization and biocomposites application A review. International Journal of Biological Macromolecules (ISSN: 0141-8130, 2016) 
6. R. Teygeler. Water hyacinth paper. Contribution to a sustainable future. Papier en Water/Paper and Water. $168-188(2000)$

7. A. Mandal, D. Chakrabarty. Isolation of nanocellulose from waste sugarcane bagasse (SCB) and its characterization. Carbohydrate Polymers. 86, $1291-1299$ (2008)

8. A. Abdulkhani, J. Hosseinzadeh, A. Ashori, S. Dadashi, Z. Takzare. Preparation and characterization of modified cellulose nanofibers reinforced polylactic acid nanocomposite. Polymer Testing. 35, 73-79 (2014)

9. S. Ummartyotin, C. Pechyen. Microcrystalline cellulose and polypropylene based composite: A simple, selective and effective material for microwavable packaging. Carbohydrate Polymers. 142, 133-140 (2016)
10. T. Kemala, E. Budianto, B. Soegiyono. Preparation and characterization of microspheres based on blend of poly (lactic acid) and poly (E-caprolactone) with poly (vinyl alcohol) as emulsifier. Arabian Journal of Chemistry. 5, 103-108 (2012)

11. M.K. Mohamad Haafiza, A. Hassana, Z. Zakariac, I.M. Inuwaa, M.S. Islamd, M. Jawaide. Properties of polylactic acid composites reinforced with oil palm biomass microcrystalline cellulose. Carbohydrate Polymers. 98, 139-145 (2013)

12. G. H. Yew, A. M. Mohd Yusof, Z. A. Mohd Ishak, U. S. Ishiaku. Water absorption and enzymatic degradation of poly (lactic acid)/rice starch composites. Polymer Degradation and Stability. 90, 488500 (2005) 